DOI: $10.1002 / \mathrm{mame}$

Article Type: Full Paper

Effects of silk degumming process on physicochemical, tensile, and optical properties of regenerated silk fibroin a

Kira Nultsch, Livia K. Bast, Muriel Näf, Salima El Yakhlifi, Nico Bruns, Oliver Germershaus*

\title{
K. N.
}

Department of Pharmaceutical Sciences, University of Basel, Klingelbergstrasse 50, 4056

Basel, Switzerland

K. N., M. N., S. E. Y., Prof. Dr. O. G.

Institute of Pharma Technology, University of Applied Sciences and Arts Northwestern

Switzerland, Gründenstrasse 40, 4132 Muttenz, Switzerland

E-mail: oliver.germershaus@fhnw.ch

L. K. B., Prof. Dr. N. B.

Adolphe Merkle Institute, University of Fribourg, Chemin des Verdiers 4, 1700 Fribourg, Switzerland

Sericin removal from silk (degumming) affects material characteristics of silk fibroin. Sodium carbonate is most commonly used for degumming, but numerous alternative methods are available. Herein, a systematic comparison of degumming methods is provided. Sodium carbonate, sodium oleate, trypsin and ionic liquid were used, and materials were characterized regarding mass loss, silk fibroin content, molecular integrity of silk fibroin, refractive index, and tensile properties. Complete degumming was achieved within 30 minutes using sodium carbonate, but resulted in significant reduction of molecular weight, shift towards less acidic charge variants and reduction of yield- and rupture force. Sodium oleate and trypsin were inefficient and negatively affected tensile properties, while ionic liquid showed good efficiency and marginal degradation of silk fibroin but also reduced yield- and rupture force. Refractive

\footnotetext{
${ }^{\text {a }}$ Supporting Information ((bold)) is available online from the Wiley Online Library or from the author.
} 
index was not affected by degumming. These results allow rational selection of the degumming method and tuning of silk fibroin properties for biomedical applications.

native silk fibroin
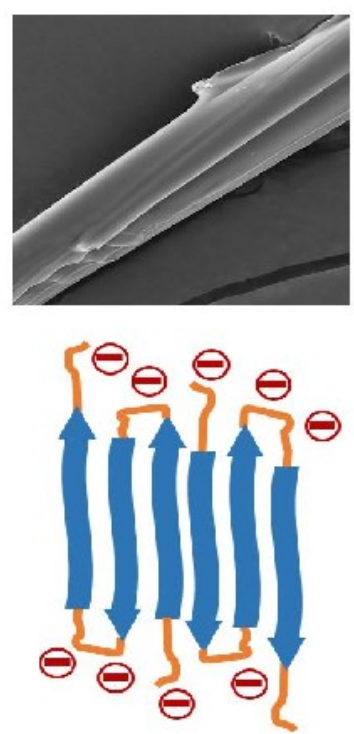

degummed silk fibroin

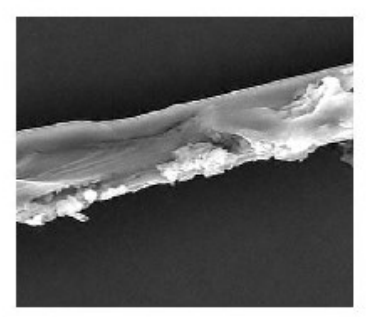

degumming

process

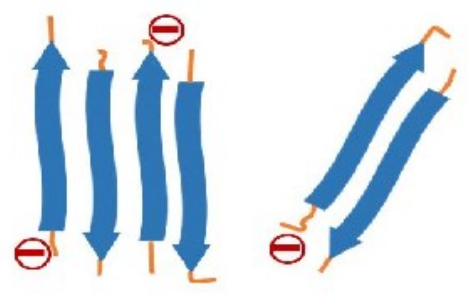




\section{Introduction}

Silk fibroin (SF), a protein produced by the mulberry silkworm Bombyx mori, has numerous advantages as scaffold for drug delivery systems, and for biomaterials. It is biocompatible, biodegradable into non-toxic products and possesses superior mechanical strength ${ }^{[1,2]}$. Not surprisingly, silk fibroin has been largely explored as a natural material for various technical and biomedical applications such as drug delivery, tissue engineering, biosensors, electronics and optics ${ }^{[3-7]}$. Silkworm silk fibers (bave) consist of a pair of filaments (brin) composed of SF which are coated and held together by the glue-like protein sericin. SF consists of a hydrophobic heavy ( $\sim 370 \mathrm{kDa}$ ) and a hydrophilic light ( $\sim 25 \mathrm{kDa})$ chain, connected by a disulfide bond. The heavy chain is formed by highly repetitive crystalline fractions, GAGAGS, GAGAGY and GAGAGVGY (Gly-X domains), and responsible for the formation of anti-parallel $\beta$-sheets ${ }^{[8]}$. For the preparation of drug delivery systems or when intended for human implantation, purification of SF is mandatory due to immunogenicity in presence of sericin ${ }^{[9]}$. This extraction and purification process (frequently referred to as degumming) is usually characterized by quite harsh conditions (elevated temperature, alkaline $\mathrm{pH}$ ), resulting not only in sericin degradation but also partial hydrolysis of fibroin ${ }^{[10]}$. Longer degumming in boiling sodium carbonate solution was reported to result in reduction of average molecular weight of $\mathrm{SF}^{[11,12]}$. While degumming using sodium carbonate solution is the most common process applied for preparation of silk fibroin for biomedical applications, various alternative degumming conditions and processes were published, e.g. degumming with citric acid ${ }^{[13]}$, urea ${ }^{[14]}$, tartaric acid ${ }^{[10]}$, different enzymes ${ }^{[15,16]}$ and ionic liquids ${ }^{[17]}$. Besides the different degumming reagents, different solvents for subsequent dissolution of SF were tested and compared, e.g. lithium bromide, Ajisawa's reagent and formic acid ${ }^{[14,18]}$ and the influence of dissolution time was studied ${ }^{[19]}$.

The degumming process conditions are known to primarily affect molecular structure of SF. Besides general reduction of average molecular weight depending on degumming time using 
sodium carbonate, it has been found that SF degradation appears to specifically occur in amorphous regions while crystalline Gly-X domains are largely unaffected ${ }^{[11,12]}$. As a result, formation of beta-sheets is virtually unaffected by degumming process duration using sodium carbonate ${ }^{[12]}$. Besides reduction of molecular weight and despite unchanged beta-sheet formation, changes in the ability to form higher order structures were observed ${ }^{[12]}$. Furthermore, degumming was shown to affect in vitro cytocompatibility. SF treated with alkali for extended durations showed inhibitory effects on fibroblast cell growth compared to fibroin taken directly from gland ${ }^{[20]}$. Additionally, the degradation profile and drug release kinetics play a key role for the use as scaffold in tissue engineering and drug delivery applications. Variation of degumming time was found to affect in vitro degradation and drug release kinetics of various model compounds ${ }^{[21]}$. Recently, we studied the impact of different degumming times on the release of differently charged high molecular weight compounds and reported that degumming not only influenced SF integrity but also SF charge distribution and hence release of charged compounds ${ }^{[12]}$. Finally, degumming influences SF tensile properties, resulting in reduction of failure strength and yield point ${ }^{[22]}$.

In summary, degumming process conditions substantially change physicochemical and mechanical properties of SF which in turn significantly affects the performance of SF as a scaffold in various biomedical applications such as drug delivery and tissue engineering. However, to our knowledge no comprehensive study on the effect of different degumming processes and process conditions has been performed for regenerated SF in the context of biomedical applications. Therefore, we herein investigate the effect of different degumming strategies such as enzymatic and alkali degumming as well as degumming using ionic liquid (IL) and process conditions on physicochemical, mechanical and optical properties of SF relevant for biomedical applications.

\section{Experimental Section}




\subsection{Materials}

Silkworm cocoons were purchased from Wollspinnerei Vetsch (Pragg-Jenaz, Switzerland). All other chemicals were ordered from Sigma Aldrich (Buchs, Switzerland).

\subsection{Methods}

\subsubsection{Silk fibroin extraction}

SF was extracted using various degumming reagents and process conditions (Table 1). In brief, SF cocoons were cut into small pieces and incubated in the respective solution at $5 \mathrm{~g} \mathrm{l}^{-1}$ under constant stirring at $300 \mathrm{rpm}$ at the temperature and time as detailed in Table 1. Sodium oleate concentration was chosen to be slightly above the critical micelle concentration. Trypsin concentration was selected based on the literature ${ }^{[15]}$. The concentration of 1-butyl-3-methylimidazolium bromide (IL) and incubation temperature were optimized to achieve maximum degumming efficiency. After degumming, SF fibers were dried at room temperature in a fume hood overnight and dissolved in Ajiwasa's reagent (calcium chloride:ethanol:water at a molar ratio of $1: 2: 8)$ at $65^{\circ} \mathrm{C}$. The solution was filtered through syringe filter with nominal pore size of $5 \mu \mathrm{m}$ (Yeti PVDF HPLC syringe filter, Infochroma AG, Zug, Switzerland) and dialyzed against purified water (Spectra/Por dialysis tubes MWCO 6-8 kDa, Spectrum Laboratories, Rancho Domingez, CA, USA). The mass of silk fibroin contained in a known volume of the solution was determined gravimetrically after evaporation of water at $65^{\circ} \mathrm{C}$ for $24 \mathrm{~h}$. For further processing, all silk fibroin solutions were adjusted to $10.0 \mathrm{~g} \mathrm{l}^{-1}$.

\subsubsection{Scanning Electron Microscopy}

The morphology of differently degummed fibers was studied by scanning electron microscopy (SEM). The fibers were sputter coated with gold and characterized with an accelerating voltage of $5 \mathrm{kV}$ using a Hitachi TM3030 plus (Hitachi, Krefeld, Germany).

\subsubsection{Mass loss}


Before and after the degumming step the dry mass of the material was determined for 4 individual samples (IL: 2 individual samples) and percentage mass loss (ML) was calculated according to Equation 1, where $m_{\text {before degumming }}$ is the dry mass before and $\mathrm{m}_{\text {after degumming }}$ the dry mass after the degumming process.

$$
M L=\frac{m_{\text {before degumming }}-m_{\text {after degumming }}}{m_{\text {before degumming }}} \times 100
$$

Degumming efficiency based on mass loss was calculated by setting mass loss observed for C120 as $100 \%$ degumming efficiency. Percentage degumming efficiency (DE) was calculated using Equation 2.

$$
D E=\frac{M L_{\text {Sample }}}{M L_{\mathrm{C} 120}} \times 100
$$

\subsubsection{Amino acid composition}

To determine the amino acid composition, degummed samples (Table 1) were hydrolyzed according to USP $41^{[23]}$. In brief, to one gram of degummed sample or sericin (Sericin Bombyx mori, order number S5201, Sigma Aldrich, Buchs, Switzerland), $200 \mu 16$ M hydrochloric acid (containing $0.5 \%$ phenol) was added and incubated at $115^{\circ} \mathrm{C}$ for $16 \mathrm{~h}$. Hydrochloric acid was removed using speed vacuum. To each sample $200 \mu 10.02 \mathrm{M}$ hydrochloric acid was added and incubated at $50{ }^{\circ} \mathrm{C}$ for 10 minutes. Standard solutions with 50, 100, 200, 300, 400, 500, 600 and $1000 \mu \mathrm{M}$ of amino acids (glycine, alanine, tyrosine, serine, glutamic acid, aspartic acid), chosen based on the significant difference in weight percentage found in sericin compared to fibroin, were prepared. Samples and standard solutions were derivatized using $70 \mu$ borate buffer (pH 8.8), $10 \mu \mathrm{l}$ sample or standard solution and $20 \mu 1$ 6-aminoquinolyl- $N$ hydroxysuccinimidyl carbamate, vortexed for $10 \mathrm{~s}$ and heated at $55^{\circ} \mathrm{C}$ for 10 minutes. Then, the samples and standards were analyzed by RP HPLC (Agilent 1260 Infinity, Agilent Technologies, Santa Clara, CA, USA; Acquity UPLC column, BEH shield RP 18, $1.7 \mu \mathrm{m}, 2.1$ x $100 \mathrm{~mm}$, Waters Corporation Milford, MA, USA) at $55{ }^{\circ} \mathrm{C}$ with a flow rate of $0.4 \mathrm{ml} \mathrm{min}{ }^{-1}$ 
(gradient steps Table 2). Buffer A consisted of a pre-prepared solution of acetonitrile, formic acid and $100 \mathrm{mM}$ ammonium formate (10:6:84), which was diluted with water (5:95). Buffer B consisted of acetonitrile and formic acid (98:2). The proportion of sericin and fibroin in the samples was calculated by setting $\mathrm{C} 120$ as $100 \%$ fibroin and sericin to $0 \%$ fibroin. Percentage degumming efficiency was calculated based on SF content (SFC) by setting cocoons to $0 \%$ degumming efficiency and C120 to $100 \%$ degumming efficiency according to Equation 3:

$$
D E=\frac{S F C_{\text {Sample }}-S F C_{\text {Cocoon }}}{S F C_{\mathrm{C} 120}-S F C_{\text {Cocoon }}} \times 100
$$

\subsubsection{Size exclusion chromatography}

The chromatographic system (Agilent 1260 Infinity, Agilent Technologies, Santa Clara, CA, USA) used for size exclusion chromatography (SEC) was equipped with a quaternary pump, auto sampler, column oven, diode array detector (DAD) and a static and dynamic light scattering detector (SLS and DLS). The separation was performed using an Agilent Bio SEC-3

column ( $3 \mu \mathrm{m}, 300 \AA$, $4.6 \times 300$ mm, Agilent Technologies, Santa Clara, CA, USA) with $0.1 \mathrm{M}$ sodium chloride as mobile phase and a flow rate of $0.3 \mathrm{ml} \mathrm{min}^{-1}$ at $30{ }^{\circ} \mathrm{C}$.

\subsubsection{Weak anion exchange chromatography}

For weak anion exchange chromatography (WAX) the same chromatographic system as for SEC was used. The separation was performed using a Bio WAX ion-exchange column ( $3 \mu \mathrm{m}$, 4.6 x 150 mm, Agilent Technologies, Santa Clara, CA, USA) with step gradients (Table 3). Buffer A consisted of $20 \mathrm{mM}$ tris-(hydroxymethyl)-aminomethan (Tris), adjusted to $\mathrm{pH} 8.5$ with hydrochloric acid. Buffer B consisted of Buffer A plus $2 \mathrm{M}$ sodium chloride. The separation was performed with a flow rate of $0.5 \mathrm{ml} \mathrm{min}^{-1}$ at $30^{\circ} \mathrm{C}$.

\subsubsection{Tensile testing}

Single silk baves were unreeled from cocoons mounted into steel frames and degummed according to Table 1. To avoid batch-to-batch variability, one cocoon was unreeled and 
samples, taken from this cocoon, were measured in triplicates. Afterwards, degummed fibers were rinsed with water and dried in a fume hood over night. Then the frames with the fibers were fixed in a Texture Analyser TA-XT2i (Stable Microsystems Ltd., Surrey, UK) with grips and the frames were opened laterally to allow pulling. Test speed was set to $0.17 \mathrm{~mm} \mathrm{~s}^{-1}$ until rupture of the fibers.

\subsubsection{Ellipsometry}

Silicon wafers $\left(2.25 \mathrm{~cm}^{2}\right.$, thickness: $675 \mu \mathrm{m}$, polished on one side from Silicon Materials, Kaufering, Germany) were washed with acetone and ethanol (ultrasound (SW 3 by Sonoswiss AG, Ramsen, Switzerland) each $15 \mathrm{~min}$ ) and cleaned with carbon dioxide snow. Overcoats of silk fibroin of various degumming times and concentrations were prepared by spin casting the fibroin solutions onto the $\mathrm{SiO}_{2}$ surface at a spin speed of $4000 \mathrm{rpm}$ for $40 \mathrm{~s}$ (WS 650-MZ23NPPB, Laurell Technologies Corporation, North Wales, USA). The samples were dried under vacuum in a desiccator over $\mathrm{CaH}_{2}$ for at least $3 \mathrm{~h}$. To induce beta-sheet formation, samples were placed in methanol for $15 \mathrm{~min}$ and again dried under vacuum in a desiccator over night.

The refractive indices of dry silk fibroin films (film thickness between $26 \pm 2 \mathrm{~nm}$ and $47 \pm 5$ $\mathrm{nm}$ ) on silicon wafers were measured using an alpha-SE Ellipsometer (J. A. Woollam, Lincoln, USA). Experimental data were modelled by the Cauchy fit for transparent films using the software Complete EASE, Version 5.19, choosing 1.50 as starting point for the fit of the refractive index of the protein. Refractive index measurements were taken at three different angles $\left(65^{\circ}, 70^{\circ}, 75^{\circ}\right)$ on three different spots on each film, and the measurements were repeated on 10 different films of each sample.

\subsubsection{Statistical analysis}

All measurements were performed in triplicates unless stated otherwise and results are presented as mean \pm standard deviation. Two-tailed Student's t-test or one-way ANOVA with 
Tukey's test (mass loss, SF content) or Dunnett's test (amino acid composition, untreated cocoons as control) was performed to identify statistical significance. Probability values of $\mathrm{p} \leq$ 0.05 were considered statistically significant.

\section{Results}

\subsection{Microscopic characterization}

Scanning electron micrographs of individual fibers after degumming were recorded to visually evaluate the removal of sericin as well as the microstructure of SF filaments (Figure 1 A-I). The native, untreated bave (Figure 1 A) showed two fibroin filaments, which are coated with and connected by sericin. After degumming using sodium carbonate (Figure 1 B-E), filaments without sericin coating were found and after extended degumming duration fibril formation at the surface of individual filaments was observed (Figure $1 \mathbf{C}$ and E). After treatment with sodium oleate (Figure 1 F) filaments were partly disconnected and spots were visible on the filament surface, likely representing sericin residues. After enzyme treatment with trypsin for two and three hours (Figure $1 \mathbf{G}$ and $\mathbf{H}$ ), the fiber appeared cracked with a puckered surface. Degumming with ionic liquid (Figure 1 I) resulted in filaments, which partly appeared to be still connected and coated with sericin, and spots were visible on the fiber surface. Figure S1 in supporting information provides additional micrographs of the samples after degumming.

\subsection{Mass loss}

SF degumming efficiency was assessed by the mass lost during the degumming process (Figure $\mathbf{1} \mathbf{J}$ and $\mathbf{K}$ ). However, it should be highlighted that mass loss during degumming may represent sericin and fibroin degradation. Mass loss may therefore be indicative of efficient removal of sericin but excessive mass loss may indicate substantial hydrolysis of fibroin. Sodium carbonate treatment resulted in overall highest mass loss and highest degumming efficiency, whereas the 
degumming with sodium oleate lead to the lowest mass loss. Within the different degumming times with sodium carbonate, treatment for 5 minutes resulted in significantly lower mass loss than longer degumming times. No statistically significant difference was observed for mass loss for C30, C60 and C120. Treatment with trypsin resulted in statistically significantly higher mass loss than sodium oleate treatment. Incubation with trypsin for 2 or 3 hours lead to similar mass loss with statistically insignificant differences between the incubation times. Ionic liquid degumming lead to statistically insignificant different mass loss as treatment with trypsin and was also similar to $\mathrm{C} 5$.

\subsection{Amino acid composition}

Amino acid composition was determined focusing on glycine, alanine, tyrosine, serine, aspartic acid and glutamic acid because the amino acid content differed significantly between in SF and sericin (Figure 2 A and B). Glycine content differed significantly between cocoon and sericin. All degummed samples showed statistically significantly higher glycine content than untreated cocoon due to partial or complete hydrolysis of sericin. Similarly, statistical significant differences in alanine content were found between untreated cocoons and sericin as well as degummed samples except OL and IL. Variation of tyrosine content between cocoons and sericin was rather small but significant. Major differences between cocoons and sericin as well as degummed samples were observed in serine content. Serine content in all degummed samples was significantly lower than in cocoons, again confirming partial or complete hydrolysis of sericin. The same was found for aspartic acid and glutamic acid.

Determination of the amino acid composition allowed calculation of fibroin content and degumming efficiency (Figure 2 C and D). Fibroin content was statistically significantly higher than in cocoons for all degummed samples except OL. No significant differences were observed for fibroin content after treatment with sodium carbonate for different durations or fibroin content after treatment with trypsin for different durations. Since fibroin content in 
untreated cocoons was already approx. $80 \%$, differences between the degumming processes are more evident if degumming efficiency is assessed (Figure $\mathbf{2} \mathbf{D}$ ). Complete degumming is observed after 30 minutes treatment with sodium carbonate. Incomplete degumming is found only after treatment with sodium oleate and to a smaller extend trypsin for 2 hours.

It should be noted, that degumming efficiency by mass loss and degumming efficiency by amino acid analysis will produce different results. In the case of degumming efficiency by mass loss the entire mass lost during degumming is assessed (i.e. sericin loss and fibroin loss). In contrast, degumming efficiency by AA analysis assesses fibroin content of the material after degumming.

\subsection{Size exclusion and weak anion exchange chromatography}

Silk cocoons showed a single peak at 5.6 minutes in size exclusion chromatography using UV detection (Figure 3 A). With longer degumming time in sodium carbonate (C5, C30, C60 and C120) the main peak became smaller and a second broad peak appeared at longer retention times (lower molecular weight). In addition, a small leading peak was observed at approx. 5.0 minutes. OL exhibited similar results compared to cocoon sample but in addition a small shoulder appeared at approx. 5.4 minutes. T120 and T180 did not show peaks at the same position as the other samples, but peaks at longer retention times were observed. IL exhibited a similar chromatogram as untreated cocoons.

Using static light scattering detection (Figure 3 B), the main peak observed by UV detection at approx. 5.6 minutes was confirmed but in addition the small leading peak at approx. 5.0 minutes was more pronounced. OL showed three peaks, the retention time of two of them was comparable to carbonate-degummed samples (peaks at 5.0 and 5.6 minutes). In addition, a new peak was observed at 5.3 minutes (potentially corresponding to the shoulder observed using UV detection). Virtually no static light scattering signals were obtained in the case of T120, T180 and IL. 
Weak anion exchange chromatography of cocoon and sodium carbonate treated samples revealed a main peak at approx. 9.1 minutes with side peak (less acidic variants) at approx. 8.4 minutes (Figure 3 C). With increasing degumming time with sodium carbonate, the main peak area was reduced and new variant peaks at approx. 8.6, 8.7 and 8.9 minutes appeared and became more prominent. OL showed a similar chromatogram as the cocoon sample with slightly more pronounced variant peaks at 8.4 minutes. Chromatograms of T120 and T180 were comparable to $\mathrm{C} 120$ with even more pronounced variants showing shorter retention times. Finally, IL only showed the variant peak at approx. 8.4 minutes.

\subsection{Tensile testing}

In Figure 4 the maximum rupture forces and yield points are displayed. Native, untreated fibers had the highest rupture force $(\sim 150 \mathrm{mN})$ and yield point $(\sim 60 \mathrm{mN})$, whereas sodium carbonate treatment resulted in significant reduction of both rupture force and yield point with increasing degumming time. No significant differences were observed between C60, C120, OL and IL. However, trypsin treatment resulted in further significant reduction of mechanical properties, especially comparing C120 with T180.

\subsection{Refractive index}

Within the margin of error of the measurements, no major variation of refractive index with degumming method and -duration were observed, neither before nor after methanol treatment to induce beta sheet formation (Figure 5). Comparing the results before and after treatment with methanol, no significant differences were observed between the two groups either.

\section{Discussion}

The degumming process, i.e. the removal of sericin from virgin silk, is one of the most critical process steps during preparation of regenerated SF, which is increasingly used as a biomaterial for tissue engineering, implantable devices, disease models and for drug delivery systems ${ }^{[24]}$. 
Depending on degumming process conditions, various material properties such as tensile strength, molecular weight distribution and micelle formation of SF are affected, ultimately affecting performance in biomedical applications such as tissue engineering and drug delivery $[12,21,22]$. Furthermore, efficient degumming is crucial with regards to the biocompatibility and immunogenicity of SF ${ }^{[11]}$. Therefore, it is necessary to completely remove sericin, while preserving or controlling SF integrity.

Numerous different processes and conditions have been published for silk fibroin extraction from virgin silk, the most common of these processes uses boiling sodium carbonate solution for degumming. Traditionally, silk textiles have been treated after weaving with alkali-free olive oil soap (Marseilles soap) at $90-98^{\circ} \mathrm{C}$ for several hours to remove sericin and resulting in reduced brittleness, improved handling and characteristic luster of silk textiles ${ }^{[25]}$. Common alternative degumming processes, especially in the context of SF extraction for biomedical applications include enzymatic degradation of sericin and, more recently, degumming using ionic liquids ${ }^{[15,17]}$.

Degumming using sodium carbonate solution efficiently hydrolyzed and removed sericin after 30 minutes as evidenced by evaluation of degumming efficiency based on mass loss (Figure 1 K) and amino acid analysis (Figure 2 D). Extending the process duration beyond 30 minutes did not significantly improve degumming efficiency based on either analysis. Published analysis of degumming efficiency showed variable results ranging from complete degumming after 5 minutes at $100^{\circ} \mathrm{C}^{[11]}$ to complete degumming after 40 minutes at $80^{\circ} \mathrm{C}^{[26]}$, presumably due to variable process conditions such as temperature, agitation and silk concentration. Increasing degumming time using sodium carbonate on the other hand substantially affected SF integrity. In agreement with previous reports, average molecular weight of SF was reduced, and molecular weight distribution increased with increasing degumming time (Figure $3 \mathbf{A}$ ) ${ }^{[11 \text {, }}$ $12,21,27,28]$. Interestingly, incubation in sodium carbonate also resulted in a shift towards less acidic charge variants with increasing degumming time (Figure $3 \mathbf{C}$ ), suggesting predominant 
degradation of the light subunit and/or C-terminal region of the heavy chain of SF ${ }^{[29]}$. This conclusion corresponds with the previous finding that beta-sheet content of SF is not significantly affected by degumming time ${ }^{[11,12]}$. Tensile testing (Figure 4 A) revealed reduction of both rupture force and yield point with increasing degumming time. The elastic behavior of silk was related to the amorphous phase as well as beta-sheet crystals ${ }^{[30]}$, while rupture force is mainly governed by failure of crystalline units ${ }^{[31]}$. Therefore, it may be hypothesized that degumming in addition to degradation of amorphous regions also reduced crystallite integrity resulting in the reduction of both yield point and rupture force ${ }^{[22]}$. Finally, changes in amino acid composition of proteins may result in small but significant differences of refractive indices ${ }^{[32]}$. However, refractive indices showed no significant changes in response to increasing degumming time (Figure 4 B), in line with recently published results ${ }^{[33]}$ and potentially due to the fact that the contribution of repetitive elements of SF heavy chain dominate refractive index. This finding contributes to the conclusion that degradation primarily affected non-repetitive regions of SF.

Oleate degumming was significantly less efficient than other methods and resulted in incomplete removal of sericin with degumming efficiency in the range of 44 to $63 \%$ (Figures $1 \mathbf{K}$ and 2 D) with remnants of sericin coating visible in micrographs (Figure 1 F). In contrast, mass losses of approx. 22 to $24 \%$ were reported for degumming using Marseille's soap at $5 \mathrm{~g}$ $1^{-1}$ or $25 \%$ of weight of the fabric at 93 or $95^{\circ} \mathrm{C}$ and 90 minutes $[34,35]$ which would be comparable to degumming efficiency achieved for C5 in the present study. While SEC and anion exchange chromatography revealed no major degradation and despite incomplete degumming, oleate treated sample showed significantly reduced yield point as well as rupture force. Degumming with Marseille's soap was reported to similarly result in pronounced strength loss, even at still incomplete degumming ${ }^{[34]}$. This observation might be explained by the contribution of sericin to tensile properties and/or by minor hydrolytic degradation of fibroin. 
Numerous alternative degumming processes applying various enzymes have been published ${ }^{[34-}$ 36]. We herein focused on trypsin, due to its wide availability and its reported high degumming efficiency ${ }^{[15]}$. However, in the present study, only moderately efficient degumming of $75 \%$ and $72 \%$ after incubation for 120 minutes and of $72 \%$ and $103 \%$ after incubation for 180 minutes was achieved based on mass loss and amino acid composition, respectively, which is in good agreement with previous findings in another study ${ }^{[37]}$. Additionally, degumming using trypsin resulted in pronounced SF degradation observed in scanning electron micrographs, SEC as well as anion exchange chromatography. Tensile testing similarly showed the lowest rupture forces and low yield points after degumming with trypsin. Based on these results it is concluded that trypsin under the conditions chosen not only degrades sericin but also fibroin, resulting in formation of protein fragments and limiting its suitability for degumming of silk for biomedical applications. This result may not be surprising in view of numerous studies which successfully used trypsin for silk fibroin digestion ${ }^{[38,39]}$.

Degumming with ILs may represent another promising degumming approach due to their specific properties, e.g. tunability, versatility and low melting point (below $100{ }^{\circ} \mathrm{C}$ ). IL consist of an inorganic ion and an organic, bulky counterion carrying a delocalized charge, which prevents the formation of a stable crystal lattice. Long alkyl chains of the organic cation, e.g. 1-butyl-3-methylimidazolium (Bmim), are able to interact with the protein, leading to participation or competition of the solvent with intra- and intermolecular protein interactions and therefore, to dissolution ${ }^{[40]}$. In the present study treatment with $[\mathrm{Bmim}] \mathrm{Br}$ resulted in similar degumming efficiency to C5, both by mass loss and amino acid analysis (Figure $\mathbf{1}$ K and 2 D), while SEC showed no major degradation of SF (Figure 3 A). However, micrographs (Figure 1 I) showed remnants of sericin on the fiber surface and tensile testing revealed substantially reduced rupture force and yield point. Reports in the literature on the effects of ILs on silk are controversial. Treatment with [Bmim]OAc resulted in visually complete dissolution of silk fibers within 4 minutes at $120^{\circ} \mathrm{C}{ }^{[41]}$ while it was reported in another study 
that only $0.7 \%$ of silk fibroin was soluble in $[\mathrm{Bmim}] \mathrm{Br}{ }^{[17]}$. Differences in water content of ILs may significantly change the dissolution properties and therefore result in different dissolution characteristics. Furthermore, silk fibers were subjected to degumming prior to incubation in ILs in one of the studies, potentially resulting in improved SF dissolution due to partial degradation induced by prior degumming. It may therefore by hypothesized that IL not only dissolved sericin quite efficiently, but also interacts with fibroin, potentially changing its molecular structure and resulting in reduced mechanical properties. Degumming of silk using ILs potentially holds great potential, but further studies will be required to identify its effects on fibroin.

\section{Conclusions}

Degumming of SF using sodium carbonate not only results in fast sericin removal after 30 minutes but also affects SF average molecular weight, molecular weight distribution, isoelectric point and mechanical properties. All these properties significantly influence the performance in various biomedical applications and may be employed for tuning of SF material characteristics for specific applications. Degumming with sodium oleate and trypsin was only moderately efficient but resulted in significant SF degradation and/or substantially reduced yield point and rupture strength. On the contrary, degumming with IL was quite efficient but requires further optimization to improve tensile properties of SF after degumming. In summary, degumming using sodium carbonate solution for 30 minutes appears the best degumming process among the studied alternatives allowing to retain SF integrity and achieve complete sericin removal.

\section{Acknowledgements}

We thank Dr. Bodo Wilts (Adolphe Merkle Institute, Fribourg, Switzerland) for scientific discussions along determination of refractive index. $\mathrm{KN}$ and $\mathrm{OG}$ gratefully acknowledge 
financial support by the Swiss National Science Foundation (SNSF) under grant number 157890. LKB and NB received funding from the European Union's Horizon 2020 research and innovation program under the Marie Skłodowska-Curie grant agreement No. 722842, and from the SNSF under grant number PP00P2_172927 and the NCCR Bio-Inspired Materials.

Keywords: silk fibroin, silk sericin, degumming, tensile testing, refractive index 
References

[1] C. Vepari, D. L. Kaplan, Prog Polym Sci 2007, 32, 991.

[2] O. Germershaus, T. Luhmann, J. C. Rybak, J. Ritzer, L. Meinel, Int Mater Rev 2015, 60, 101.

[3] C. B. Borkner, M. B. Elsner, T. Scheibel, Acs Appl Mater Inter 2014, 6, 15611.

[4] B. W. Zhu, H. Wang, W. R. Leow, Y. R. Cai, X. J. Loh, M. Y. Han, X. D. Chen, Adv Mater 2016, 28, 4250.

[5] J. Melke, S. Midha, S. Ghosh, K. Ito, S. Hofmann, Acta Biomater 2016, $31,1$.

[6] L. D. Koh, Y. Cheng, C. P. Teng, Y. W. Khin, X. J. Loh, S. Y. Tee, M. Low, E. Y. Ye, H.

D. Yu, Y. W. Zhang, M. Y. Han, Progress in Polymer Science 2015, 46, 86.

[7] F. Mottaghitalab, M. Farokhi, M. A. Shokrgozar, F. Atyabi, H. Hosseinkhani, Journal of Controlled Release 2015, 206, 161.

[8] C.-Z. Zhou, F. Confalonieri, M. Jacquet, R. Perasso, Z.-G. Li, J. Janin, Proteins: Structure, Funtion, and Bioinformatics 2001, 44, 119.

[9] B. Panilaitis, G. H. Altman, J. S. Chen, H. J. Jin, V. Karageorgiou, D. L. Kaplan, Biomaterials 2003, 24, 3079.

[10] G. Freddi, G. Allera, G. Candiani, Journal of the Society of Dyers and Colourists 1996, $112,191$.

[11] L. S. Wray, X. Hu, J. Gallego, I. Georgakoudi, F. G. Omenetto, D. Schmidt, D. L. Kaplan, Journal of biomedical materials research. Part B, Applied biomaterials 2011, 99, 89.

[12] K. Nultsch, O. Germershaus, Eur J Pharm Sci 2017, 106, 254.

[13] M. R. Khan, M. Tsukada, Y. Gotoh, H. Morikawa, G. Freddi, H. Shiozaki, Bioresource technology 2010, 101, 8439.

[14] H.-Y. Wang, Y.-Q. Zhang, Soft Matter 2013, 9, 138.

[15] M. N. Padamwar, A. P. Pawar, J Sci Ind Res India 2004, 63, 323.

[16] G. Freddi, R. Mossotti, R. Innocenti, Journal of Biotechnology 2003, 106, 101.

[17] D. M. Phillips, L. F. Drummy, D. G. Conrady, D. M. Fow, R. R. Naik, M. O. Stone, P. C.

Trulove, H. C. De Long, R. A. Mantz, Journal of the American Chemical Society 2004, 126, 14350 .

[18] H. J. Cho, C. S. Ki, H. Oh, K. H. Lee, I. C. Um, Int J Biol Macromol 2012, 51, 336.

[19] J. Nam, Y. H. Park, Journal of Applied Polymer Science 2001, 81, 3008.

[20] K. Tsubouchi, H. Nakao, Y. Igarashi, Y. Takasu, H. Yamada, Journal of Insect Biotechnology and Sericology 2003, 72, 65.

[21] E. M. Pritchard, X. Hu, V. Finley, C. K. Kuo, D. L. Kaplan, Macromol Biosci 2013, 13, 311.

[22] P. Jiang, H. Liu, C. Wang, L. Wu, J. Huang, C. Guo, Materials Letters 2006, 60, 919.

[23] "Scaffold Silk FIbroin", in United States Pharmacopeia 41.

[24] D. N. Rockwood, R. C. Preda, T. Yucel, X. Q. Wang, M. L. Lovett, D. L. Kaplan, Nat Protoc 2011, 6, 1612.

[25] T. Scheibel, H. Zahn, A. Krasowski, "Silk", in Ullmann's Encyclopedia of Industrial Chemistry, 2016.

[26] M. Sah, K. Pramanik, Proceedings of 2010 International Conference on Biotechnology and Food Science (Icbfs 2010) 2010, 206.

[27] V. Trefiletti, G. Conio, F. Pioli, B. Cavazza, A. Perico, E. Patrone, Makromol Chem 1980, $181,1159$.

[28] H. Yamada, H. Nakao, Y. Takasu, K. Tsubouchi, Mat Sci Eng C-Bio S 2001, 14, 41.

[29] M. S. Zafar, D. J. Belton, B. Hanby, D. L. Kaplan, C. C. Perry, Biomacromolecules 2015, $16,606$.

[30] I. Krasnov, I. Diddens, N. Hauptmann, G. Helms, M. Ogurreck, T. Seydel, S. S. Funari, M. Muller, Phys Rev Lett 2008, 100. 
[31] M. Cetinkaya, S. B. Xiao, B. Markert, W. Stacklies, F. Grater, Biophysical Journal 2011, $100,1298$.

[32] T. L. McMeekin, M. L. Groves, N. J. Hipp, "Refractive Indices of Amino Acids, Proteins, and Related Substances", in Amino Acids and Serum Proteins, AMERICAN CHEMICAL SOCIETY, 1964, p. 54.

[33] G. Perotto, Y. J. Zhang, D. Naskar, N. Patel, D. L. Kaplan, S. C. Kundu, F. G. Omenetto, Appl Phys Lett 2017, 111.

[34] M. Arami, S. Rahimi, L. Mivehie, F. Mazaheri, N. M. Mahmoodi, Journal of Applied Polymer Science 2007, 106, 267.

[35] M. L. Gulrajani, R. Agarwal, A. Grover, M. Suri, Indian J Fibre Text 2000, 25, 69.

[36] M. L. Gulrajani, S. V. Gupta, A. Gupta, M. Suri, Indian Journal of Fibre and Textile Research 1996, 21, 270.

[37] S. V. More, H. B. Khandelwal, M. A. Joseph, R. S. Laxman, J Nat Fibers 2013, 10, 98.

[38] K. Tanaka, N. Kajiyama, K. Ishikura, S. Waga, A. Kikuchi, K. Ohtomo, T. Takagi, S. Mizuno, Bba-Protein Struct M 1999, 1432, 92.

[39] K. Yamaguchi, Y. Kikuchi, T. Takagi, A. Kikuchi, F. Oyama, K. Shimura, S. Mizuno, J Mol Biol 1989, $210,127$.

[40] C. Schroder, Top Curr Chem (Cham) 2017, 375, 25.

[41] J. Y. Chen, K. Vongsanga, X. G. Wang, N. Byrne, Materials 2014, 7, 6158. 


\section{Figures}
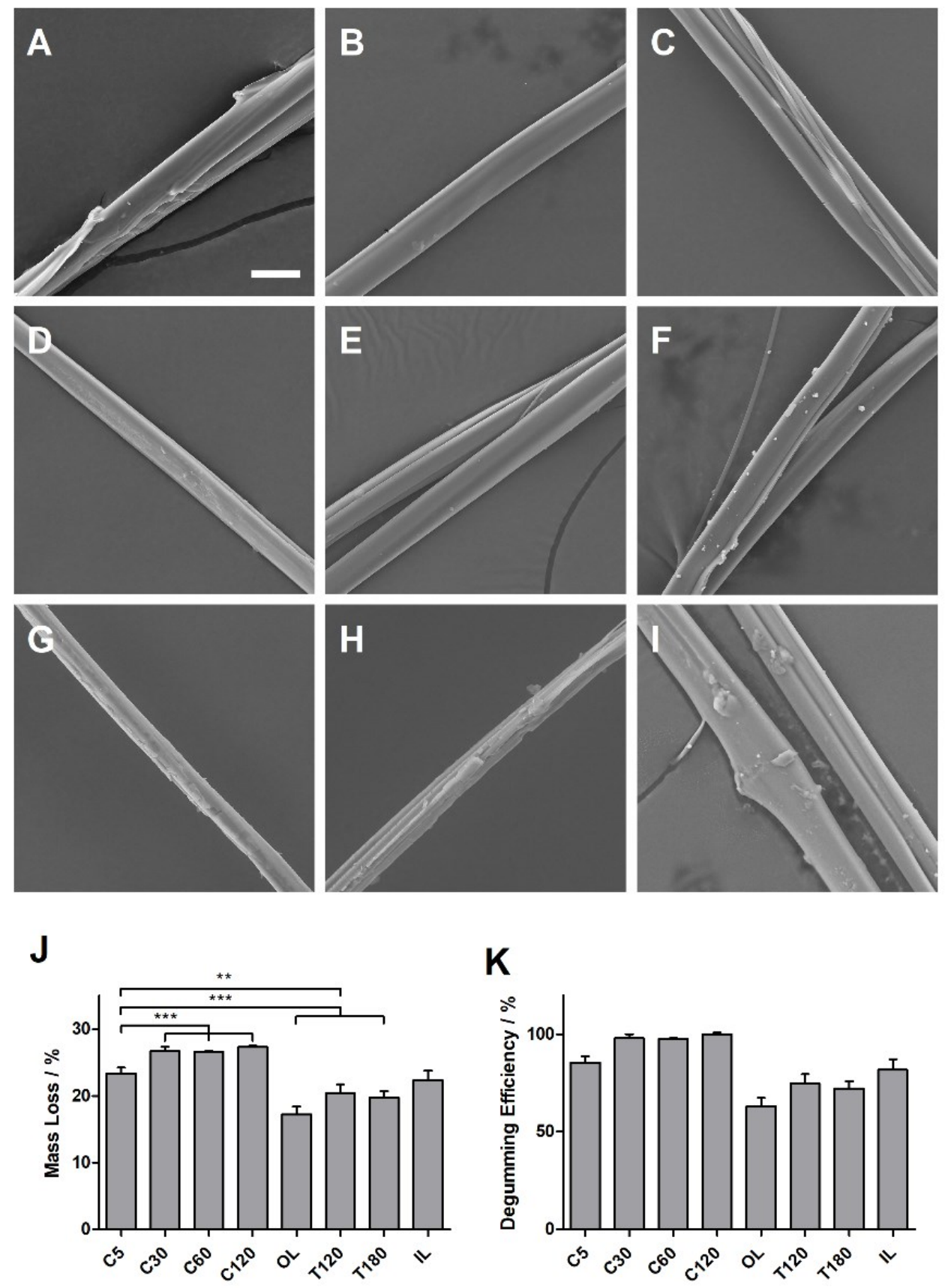

Figure 1. (A-I) Scanning electron micrographs of native and degummed fiber samples. (A) native silk fiber, (B) C5, (C) C30, (D) C60, (E) C120, (F) OL, (G) T120, (H) T180 and (I) IL. Bar in (A) represents $20 \mu \mathrm{m}$ and applies to all micrographs. (J) Mass loss during degumming process and $(\mathrm{K})$ degumming efficiency calculated based on mass loss. Asterisks indicate significance level: $* * \mathrm{p} \leq 0.01 ; \mathrm{p} \leq 0.001$. 

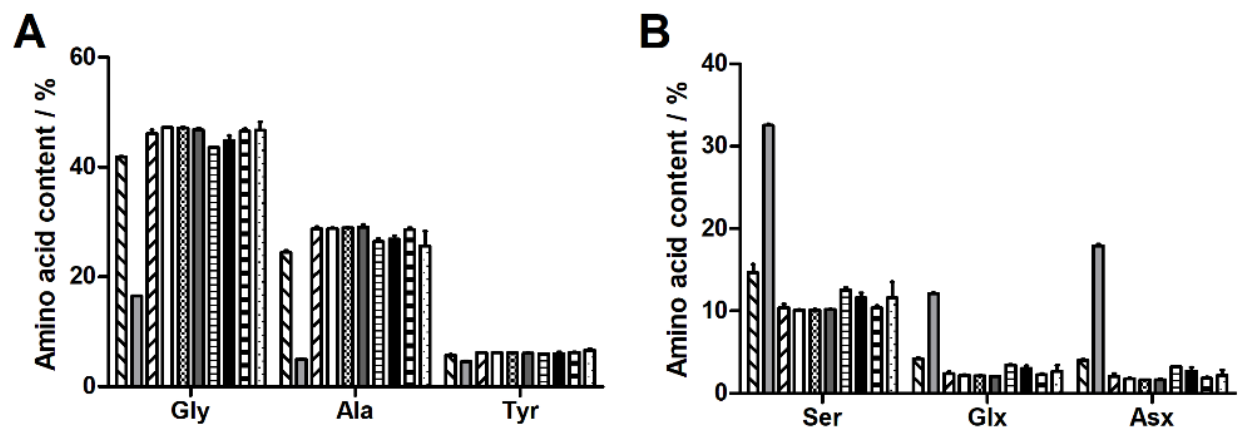

$$
\begin{aligned}
& \text { Cocoons } \square \text { Sericin } \mathbb{C 5} \square \mathrm{C} \text { C30 } \\
& \text { ख } 600 \text { C120 घ OL }
\end{aligned}
$$

C

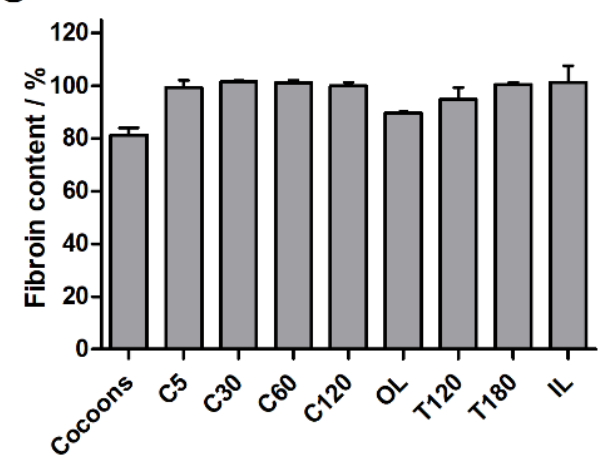

D

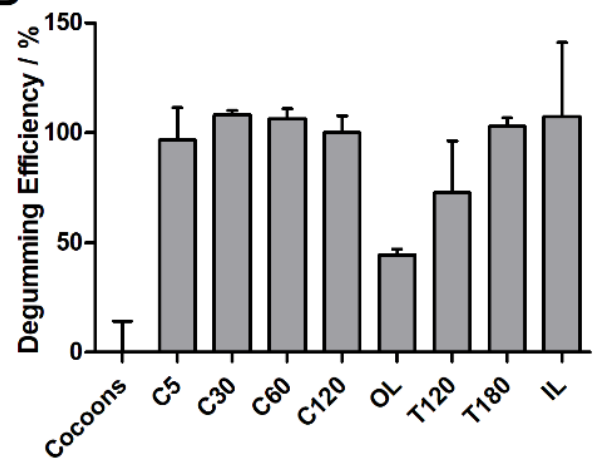

Figure 2. Amino acid content for (A) glycine, alanine, tyrosine and (B) serine, aspartic acid and glutamic acid of cocoons, sericin and degummed samples. Fibroin content (C) and degumming efficiency (D) of cocoons and samples as calculated based on amino acid composition analysis. 

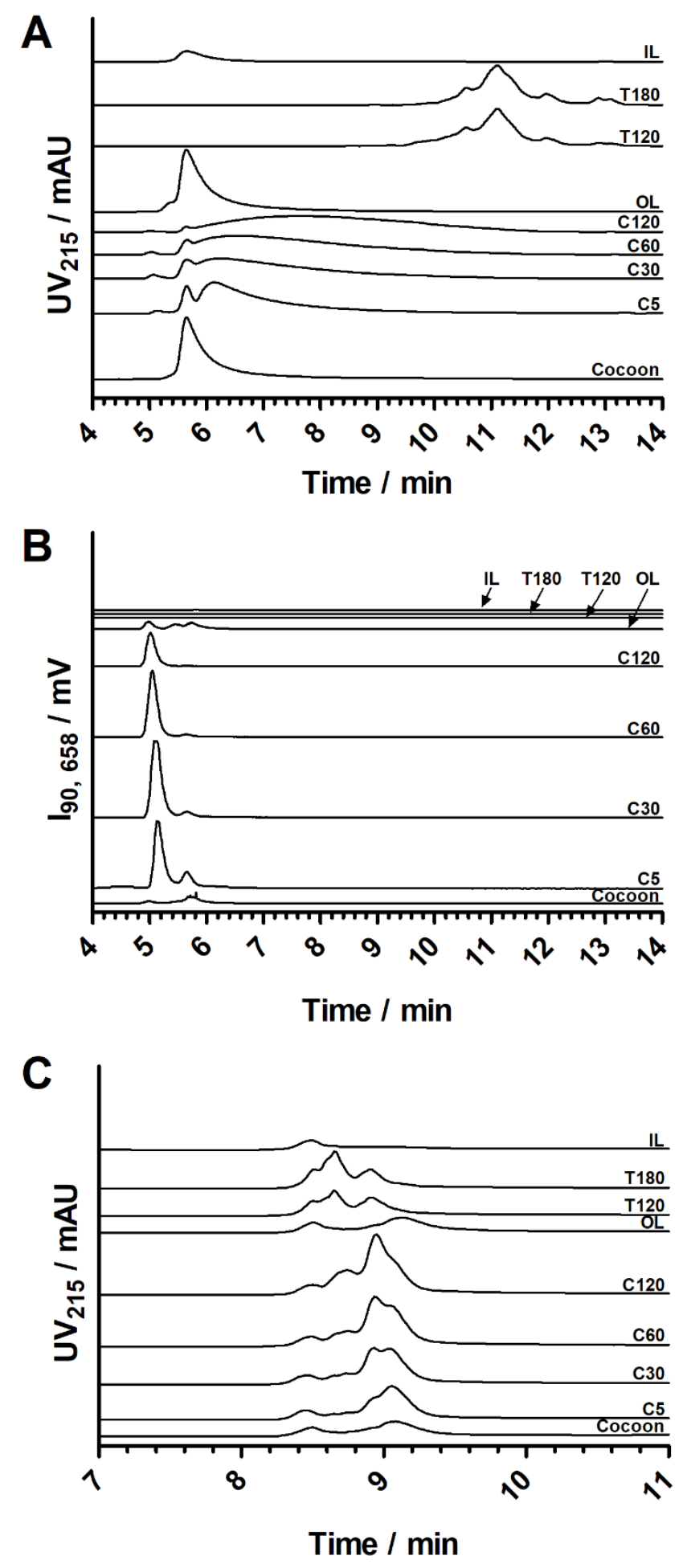

Figure 3. Chromatography of cocoons, C5, C30, C60, C120, OL, T120, T180 and IL (traces from bottom to top in each panel). Size exclusion chromatography with (A) UV detection at $215 \mathrm{~nm}$ and (B) light scattering detection at $90^{\circ}$. (C) Weak anion exchange chromatography with UV detection at $215 \mathrm{~nm}$. 


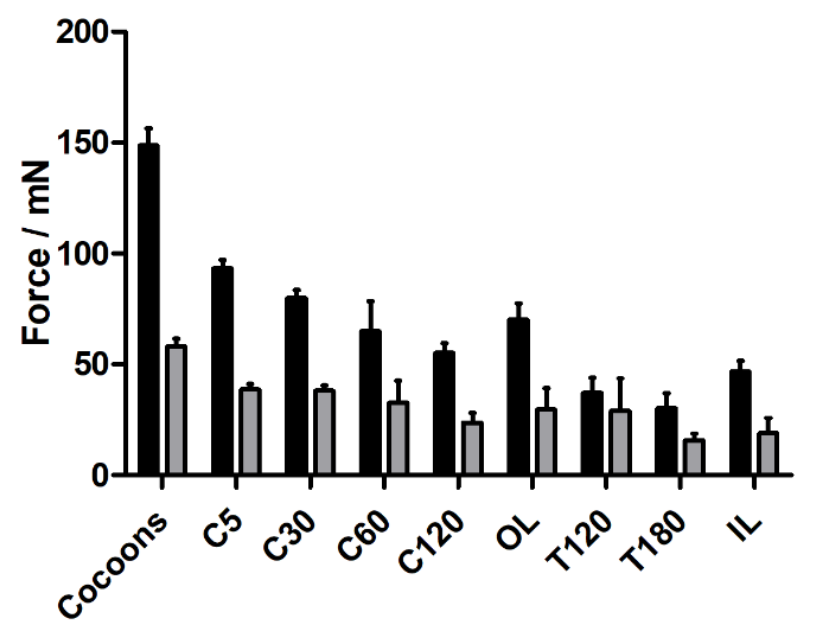

Figure 4. Tensile testing of native silk and differently degummed samples: rupture force (black bars) and yield point (grey bars).

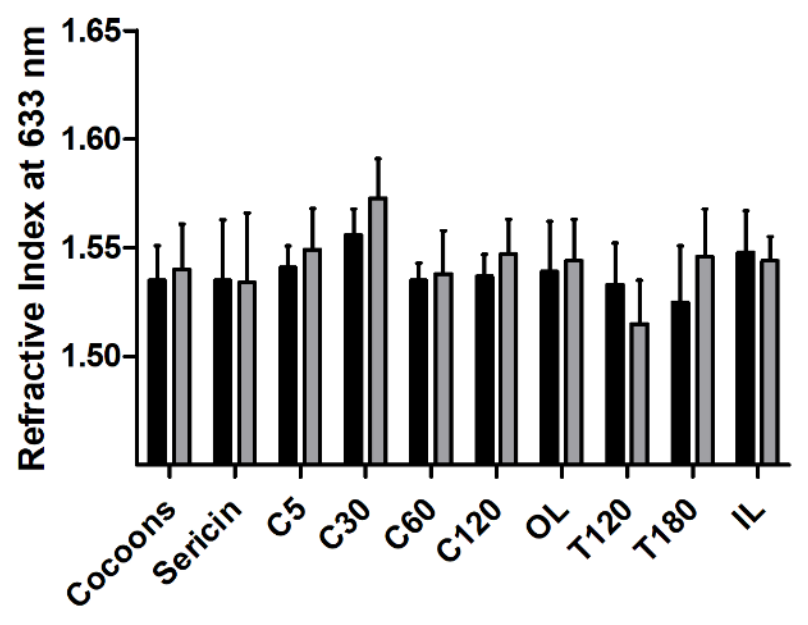

Figure 5. Refractive indices of native silk, sericin and differently degummed samples before (black bars) and after (grey bars) treatment with methanol. 


\section{Tables}

Table 1. Degumming reagents and process conditions

\begin{tabular}{|c|c|c|c|c|c|c|c|c|}
\hline Sample & C5 & C30 & C60 & C120 & OL & T120 & T180 & IL \\
\hline $\begin{array}{l}\text { Degumming } \\
\text { reagent }\end{array}$ & $\begin{array}{l}0.02 \mathrm{M} \\
\text { Sodium } \\
\text { carbonat } \\
\mathrm{e}\end{array}$ & $\begin{array}{l}0.02 \mathrm{M} \\
\text { Sodium } \\
\text { carbonat } \\
\mathrm{e}\end{array}$ & $\begin{array}{l}0.02 \mathrm{M} \\
\text { Sodium } \\
\text { carbonat } \\
\mathrm{e}\end{array}$ & $\begin{array}{l}0.02 \mathrm{M} \\
\text { Sodium } \\
\text { carbonat } \\
\mathrm{e}\end{array}$ & $\begin{array}{l}1 \% \\
\text { Sodium } \\
\text { oleate in } \\
\text { water, } \\
\mathrm{pH} 7{ }^{1}\end{array}$ & $\begin{array}{l}1 \% \\
\text { Trypsin } \\
\text { in } 67 \\
\text { mM } \\
\text { phospha } \\
\text { te buffer } \\
\text { pH } 8\end{array}$ & $\begin{array}{l}1 \% \\
\text { Trypsin } \\
\text { in } 67 \\
\text { mM } \\
\text { phospha } \\
\text { te buffer } \\
\text { pH } 8\end{array}$ & $\begin{array}{l}90 \% 1- \\
\text { butyl-3- } \\
\text { methyl- } \\
\text { imidazoli } \\
\text { um } \\
\text { bromide } \\
\text { in water }\end{array}$ \\
\hline $\begin{array}{l}\text { Degumming } \\
\text { time / } \min \end{array}$ & 5 & 30 & 60 & 120 & 60 & 120 & 180 & 420 \\
\hline $\begin{array}{l}\text { Temperature } \\
/{ }^{\circ} \mathrm{C}\end{array}$ & 100 & 100 & 100 & 100 & 90 & 37 & 37 & 85 \\
\hline
\end{tabular}

${ }^{1}$ Sodium oleate was selected as a chemically defined surrogate for Marseille soap

Table 2. RP HPLC step gradients.

\begin{tabular}{lll}
\hline Time / min & Buffer A / \% & Buffer B / \% \\
\hline 0 & 99.9 & 0.1 \\
0.54 & 99.9 & 0.1 \\
5.74 & 90.9 & 9.1 \\
7.74 & 78.8 & 21.2 \\
8.04 & 40.4 & 59.6 \\
8.64 & 40.4 & 59.6 \\
8.73 & 99.9 & 0.1 \\
9.5 & 99.9 & 0.1
\end{tabular}

Table 3. Weak anion exchange chromatography step gradients.

\begin{tabular}{lll}
\hline Time / $\mathbf{m i n}$ & Buffer A / \% & Buffer B / \% \\
\hline 5 & 100 & 0 \\
5.01 & 80 & 20 \\
10 & 80 & 20 \\
10.01 & 60 & 40 \\
15 & 60 & 40 \\
15.01 & 40 & 60 \\
20 & 40 & 60 \\
20.01 & 0 & 100
\end{tabular}


The degumming of virgin silk significantly influences the physicochemical properties and material characteristics of resulting silk fibroin. The efficiency of different degumming methods and their effect on mass loss, silk fibroin content, molecular weight and charge as well as on tensile and optical properties is investigated to allow rational process selection for biomedical applications.

Kira Nultsch, Livia K. Bast, Muriel Näf, Salima El Yakhlifi, Nico Bruns, Oliver Germershaus* Effects of silk degumming process on physicochemical, tensile, and optical properties of regenerated silk fibroin

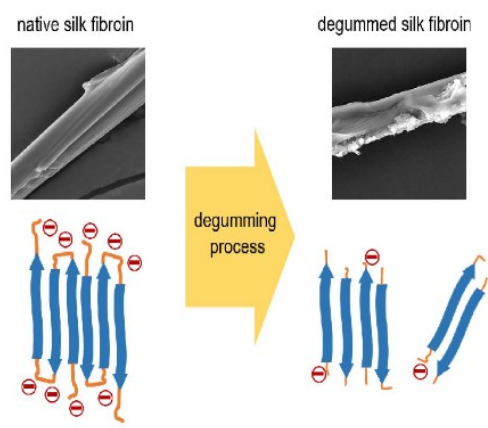


Copyright WILEY-VCH Verlag GmbH \& Co. KGaA, 69469 Weinheim, Germany, 2013.

\section{Supporting Information}

for Macromol. Mater. Eng., DOI: 10.1002/mame.2018\#\#\#\#\#

Effects of silk degumming process on physicochemical, tensile, and optical properties of regenerated silk fibroin

Kira Nultsch, Livia K. Bast, Muriel Näf, Salima El Yakhlifi, Nico Bruns, Oliver Germershaus*
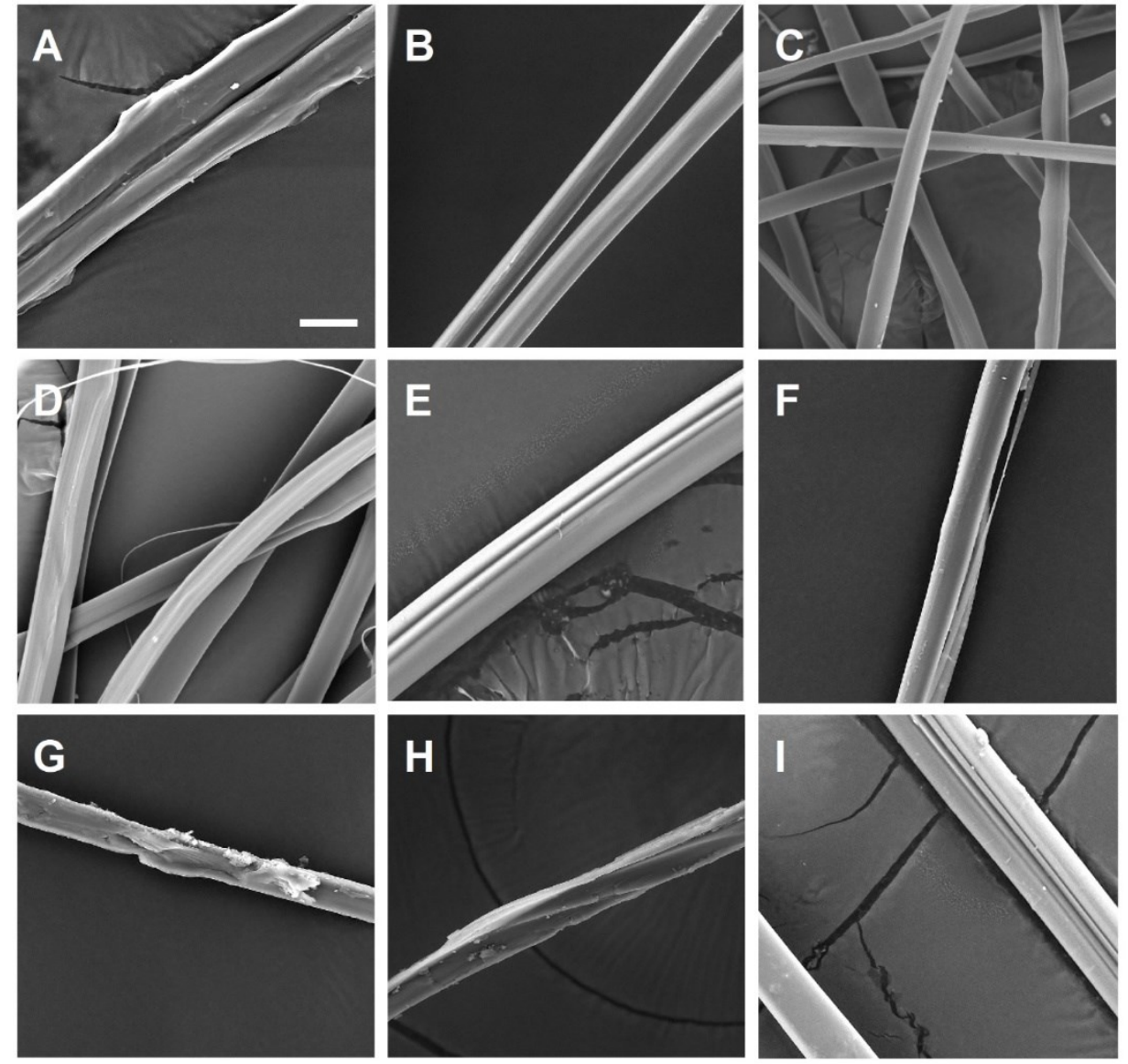

Figure S1. Scanning electron micrographs of native and degummed fiber samples. (A) native silk fiber, (B) C5, (C) C30, (D) C60, (E) C120, (F) OL, (G) T120, (H) T180 and (I) IL. Bar in (A) represents $20 \mu \mathrm{m}$ and applies to all micrographs. 